\title{
Médiévales
}

Langues, Textes, Histoire

78 | printemps 2020

Moyen Âge en séries

\section{Les séries télévisées, entre Moyen Âge et médiévalisme}

\section{Alban Gautier et Laurent Vissière}

\section{(2) OpenEdition}

1 Journals

Édition électronique

URL : https://journals.openedition.org/medievales/10722

DOI : 10.4000/medievales. 10722

ISSN : $1777-5892$

Éditeur

Presses universitaires de Vincennes

\section{Édition imprimée}

Date de publication : 20 août 2020

Pagination : 5-12

ISBN : 978-2-37924-093-5

ISSN : 0751-2708

\section{Référence électronique}

Alban Gautier et Laurent Vissière, «Les séries télévisées, entre Moyen Âge et médiévalisme »,

Médiévales [En ligne], 78 I printemps 2020, mis en ligne le 02 janvier 2022, consulté le 22 avril 2022.

URL : http://journals.openedition.org/medievales/10722; DOI : https://doi.org/10.4000/medievales 10722 


\author{
Alban Gautier, Laurent Vissière
}

\title{
Les séries télévisées, entre Moyen Âge et médiévalisme
}

Les séries télévisées occupent - le fait est bien connu - une place croissante dans les pratiques culturelles et de divertissement de nos contemporains, et nombre d'entre elles concernent la période médiévale. Il n'est donc pas étonnant qu'au sein des sciences humaines et sociales et des études littéraires, l'intérêt pour les séries historiques, notamment médiévales, se soit affûté au cours de ces dernières années ${ }^{1}$. Le présent numéro de Médiévales, né d'un appel à communications lancé au printemps $2018^{2}$, propose d'aborder ce phénomène, dans une partie de sa prodigieuse diversité, à travers neuf panoramas thématiques ou études de cas, menés par douze chercheurs : leurs origines, leur statut et leurs domaines de recherche - de la France à l'Italie, des études médiévales aux media studies - reflètent la richesse et la variété des approches que suscitent ces productions auprès de spécialistes qui, sous un apparent paradoxe, sont aussi des amateurs. Ces différentes contributions s'intègrent à ce champ de recherche en plein essor que constitue désormais le médiévalisme ${ }^{3}$.

1. On citera, parmi une production de plus en plus abondante, M. PAGÈs et K. KINANE éd., The Middle Ages on Television. Critical Essays, Jefferson (NC), 2015, et T. BRERO et S. FARRÉ éd., The Historians. Saison 1. Les séries TV décryptées par les historiens, Chêne-Bourg (Genève), 2017 : il convient de noter qu'aucun de ces deux volumes ne porte exclusivement sur les séries d'inspiration médiévale ; ainsi, la « saison 2 » de The Historians, parue en 2018, n'analyse aucune série se rapportant au Moyen Âge. À ces deux volumes collectifs, il faut ajouter de nombreux articles ou chapitres portant sur telle ou telle production.

2. À l'occasion de ce numéro, la revue s'est prêtée pour la première fois à cet exercice, qui s'est avéré fructueux (vingt-quatre propositions ont été reçues) et qui ne manquera pas d'être reproduit à l'avenir.

3. Sur le médiévalisme du début du $\mathrm{XXI}^{\mathrm{e}} \mathrm{s}$. et son intérêt pour les productions et pratiques culturelles populaires et médiatiques d'inspiration médiévale, voir D. MATTHEWS, Medievalism. A Critical History, Cambridge, 2015, p. XII. 
Par son format, le présent numéro ne prétend en aucun cas à l'exhaustivité. Les productions analysées dans les articles suivants sont de nature très variée. On trouvera des séries télévisées au sens strict ou des œuvres produites pour des plates-formes de vidéo à la demande. Il peut s'agir de séries «patrimoniales » comme Thierry la Fronde (1963-1966) ou d'œuvres plus récentes (la diffusion de certaines est toujours en cours), et même si la majorité des œuvres analysées dans les articles appartiennent aux deux dernières des quatre périodes récemment dégagées par Marjolaine Boutet $^{4}$ - le « nouvel âge d'or des séries télévisées » (des années 1980 à la première décennie du XXI ${ }^{\mathrm{e}}$ siècle) et l'âge de la "post-télévision » marqué par les nouveaux usages de streaming, de téléchargement et de vidéo à la demande (depuis 2010) -, les deux périodes antérieures - le «premier âge d'or » des années 1950 et l' «âge classique » des années 1960 et 1970 - ne sont pas entièrement négligées. Les séries étudiées sont produites en France, dans le monde anglophone ou dans d'autres aires culturelles. Certaines ont connu un succès mondial, comme Game of Thrones (2011-2019), d'autres sont beaucoup plus confidentielles.

Cela dit, malgré cette grande variété, toutes les œuvres retenues répondent à quatre critères. Le premier est un critère de sérialité, qui peut aussi bien caractériser une mini-série de quatre ou cinq épisodes qu'un récit-fleuve dont la diffusion se déroule sur plusieurs années. Il convient de noter ici que les œuvres peuvent tenir du feuilleton (où une narration unifiée et continue se déploie sur plusieurs épisodes) ou de la série stricto sensu (où, à l'intérieur d'un univers cadre, chaque épisode raconte une nouvelle histoire), sans oublier les innombrables formes intermédiaires (« feuilleton sérialisant », « série feuilletonnante », etc.) ${ }^{5}$. En outre, la plupart de ces séries partagent une architecture à un ou deux niveaux, liée aux conditions de production et de programmation : même si les termes utilisés par les showrunners et les critiques sont d'une grande variété, elles comprennent en général un certain nombre d' « épisodes », répartis ou non en « saisons » 6 .

4. M. Boutet, «Histoire des séries télévisées », dans S. SEPUlChre éd., Décoder les séries télévisées, Louvain-la-Neuve, 2017, p. 11-47.

5. Sur cette distinction, et bien que de nombreuses productions « brouillent les frontières entre les deux territoires », voir N. NEL, « Téléfilm, feuilleton, série, saga, sitcom, soap opera, telenovela : quels sont les éléments clés de la sérialité ? », CinémAction, 57 (1990), p. 6266 ; à compléter par S. BENASSI, «Sérialité(s) », dans S. SEPULCHRE éd., Décoder les séries télévisées, p. 75-105 (p. 80). La distinction rejoint (mais seulement en partie) celle opérée par J.-P. EsQUenAZI, Les Séries télévisées. L'avenir du cinéma ?, Paris, 2010, p. 104-105, entre séries « évolutives » et «immobiles ».

6. C'est pourquoi, dans les pages qui suivent, et sauf cas particulier (les «livres» de la série Kaamelott, les «feuilletons » de la série Doctor Who qui, à l'intérieur d'une même saison, contiennent chacun plusieurs épisodes), les références à un épisode précis sont données sous une forme normalisée SX/EY : ainsi la mention «Vikings, S2/E9 » désigne le $9^{\mathrm{e}}$ épisode de la $2^{\mathrm{e}}$ saison de la série Vikings. 
Les deux critères suivants précisent l'identification de nos séries comme des « fictions à la chaîne », pour reprendre l'expression de Matthieu Letourneux ${ }^{7}$. D'une part, toutes les séries retenues ont été produites pour être regardées sur le petit écran (critère 2) : le cinéma au sens strict est exclu du champ de ces études. De fait, alors que le cinéma avait été pendant des décennies un des réceptacles privilégiés du discours médiévaliste ${ }^{8}$, celui-ci semble, depuis le début de ce siècle, s'être déplacé majoritairement vers la télévision ${ }^{9}$. Même si chaque épisode dure en général moins longtemps qu'un film de cinéma, la narration de ces séries se déploie en réalité sur un temps beaucoup plus long qu'au grand écran. Ainsi, malgré leur format de trois minutes et trente seconde par épisode, les quatre premières saisons de la série française Kaamelott (2005-2006) représentent près de vingt-quatre heures de visionnage. On dépasse les soixante heures pour les huit saisons de Game of Thrones. Cela permet aux concepteurs de ces séries de créer des univers plus denses, où le décor, les costumes, les accessoires et même l'arrièreplan intellectuel ou religieux revêtent une grande importance. D'autre part, aucune des séries retenues ne relève du genre du documentaire ou du docufiction (critère 3) : que leur scénario reprenne une trame historique ou qu'il soit entièrement fictionnel, qu'elles aient ou non une prétention didactique, les productions ici analysées se présentent comme des récits romanesques plutôt que comme des sources d'information sur une période historique. Ces deux critères signifient que même les séries dont les prétentions en termes de reconstitution historique sont les plus élevées peuvent et doivent être décrites comme des fictions, toujours caractérisées par ce qui fait la double spécificité du genre : le «monde fictionnel généreux » qui, dans la longue durée du récit, multiplie les personnages, les points de vue et construit ce que Jean-Pierre Esquenazi a appelé une « encyclopédie fictionnelle », mais aussi ce « goût de l'intime », ce souci de nourrir la narration en offrant au spectateur « le spectacle de l'intimité d'autrui ${ }^{10}$ ».

Quatrième et dernier critère : toutes les séries retenues sont « médiévalisantes », c'est-à-dire qu'elles se rapportent d'une manière ou d'une autre au Moyen Âge. Il peut d'abord s'agir de mettre en scène un personnage historique ou un événement pris au sein du millénaire médiéval. L'éventail

7. M. LETOURNEUX, Fictions à la chaîne. Littératures sérielles et culture médiatique, Paris, 2017 : l'ouvrage porte principalement sur les séries de « romans populaires », mais l'analyse peut être élargie à de nombreuses autres formes, comme l'auteur lui-même l'affirme et l'esquisse.

8. Voir l'étude désormais classique de F. AMY DE LA BRETÈQUE, L'Imaginaire médiéval dans le cinéma occidental, Paris, 2004 ; voir aussi ID., Le Moyen Âge au cinéma. Panorama historique et artistique, Paris, 2015.

9. Ce constat est proposé par M. PAGÈs et K. KINANE, «Introduction : Television Medievalisms », dans EAED. éd., The Middle Ages on Television..., p. 1-11 (p. 1).

10. J.-P. ESQUENAZI, Les Séries télévisées..., p. 158-160. 
chronologique et géographique est alors aussi large que le Moyen Âge luimême : la guerre de Cent Ans de Thierry la Fronde, étudiée par Bernard Papin ; le calife 'Umar et les débuts de l'Islam dans la production qatarosaoudienne 'Umar ibn al-Khaț̣āb (2012), présentée par Enki Baptiste et Hassan Bouali ; le pays de Galles au temps d'Édouard II, théâtre de l'unique saison de The Bastard Executioner (2015), analysée par Yohann Chanoir ; ou encore la désormais célèbre série canado-irlandaise Vikings (2013-2020), à laquelle plusieurs contributions font référence. Mais les séries peuvent aussi adapter des œuvres ou des univers romanesques du Moyen Âge : la matière de Bretagne se prête particulièrement bien à ce genre de réécritures, comme le montre Aude Mairey, qui traite de la série Merlin produite par la BBC (2008-2012). D'autres productions peuvent s'appuyer sur des œuvres plus récentes, par exemple des romans historiques ayant pour cadre le Moyen Âge, à l'instar de la série britannique The Last Kingdom (2015-...), adaptée du cycle des Saxon Stories du romancier Bernard Cornwell (2004...) - elle est présente dans les articles de Pierre-Brice Stahl, de MarieEmmanuelle Torres, et de Riccardo Facchini et Davide Iacono. Ces séries que l'on peut appeler «historiques » se réfèrent de manière claire au Moyen Âge des médiévistes, mais prennent souvent de grandes libertés avec l'histoire, par parti pris, ignorance ou manque de crédits. Leur degré d'historicité s'avère de fait extraordinairement variable. Dans la plupart des cas, mieux vaut éviter de les regarder avec les yeux d'un médiéviste trop endurci. Il convient d'ailleurs de rappeler que l'anachronisme fait pleinement partie de la culture médiévale et que les lecteurs de Chrétien de Troyes ou les commanditaires de manuscrits enluminés ne se sont jamais offusqués de voir les héros arthuriens représentés en chevaliers du XII ${ }^{\mathrm{e}}$ ou du XIV ${ }^{\mathrm{e}}$ siècle.

D'autres séries médiévalisantes n'entretiennent pour leur part aucun rapport explicite avec l'histoire du Moyen Âge. Pour les réalisateurs et leur public, un certain univers de fantasy, à la fois fantastique et fantaisiste, est reconnu comme tout aussi «moyenâgeux » que la période historique éponyme ${ }^{11}$ - une tendance déjà fort présente dans le roman, le cinéma, la bande dessinée et, plus récemment, le jeu vidéo. De fait, l'identification d' « un » Moyen Âge se passe aisément de référent historique précis : la mise en scène de lugubres châteaux et de villages crasseux, voire de landes et de forêts, suffit au décor. Dans un univers fruste et violent, on se bat à l'arme blanche et le fracas des tôles froissées, à la guerre ou au tournoi, domine le paysage sonore ${ }^{12}$. L'emploi d'un vocabulaire archaïsant, soit trivial, soit

11. Sur les rapports de ce genre à la référence médiévale, voir A. BESSON, La Fantasy, Paris, 2007, p. 80-84 et 154-157 ; E. Poulain-GAUTRET, « Moyen Âge », dans A. BESSON éd., Dictionnaire de la fantasy, Paris, 2018, p. 262-269.

12. Sur le paysage sonore médiévalisant, voir L. HABLOT et L. VISSIÈRE, « Introduction », dans EID. éd., Les Paysages sonores du Moyen Âge à la Renaissance, Rennes, 2015, p. 9-15. 
courtois, mais toujours de fantaisie, donne une légère touche "littéraire » à l'ensemble. Il arrive même que le « Moyen Âge » se réduise à la mise en scène d'une «culture vaguement préindustrielle, que nous sommes censés reconnaître comme plus ou moins médiévale mais qui n'est pas clairement désignée comme telle ${ }^{13} \gg$; or le spectateur identifie ordinairement ces signes comme «médiévaux » ou «moyenâgeux », même quand ils sont relativement ténus et peu nombreux. La présence d'animaux fabuleux et le recours à la magie sont fréquents et n'ont rien de choquant, puisqu'on redonne vie - certes en le réinventant - à tout un imaginaire parfaitement médiéval. Ainsi, même s'il existe tout autant dans les traditions antiques ou extrême-orientales, le dragon, analysé ici par Lucie Herbreteau, est devenu un de ces signes de médiévalité. L'une des meilleures preuves de l'efficacité de ces clichés est leur reprise dans les productions comiques : le fait même qu'on puisse, comme dans Norsemen (2016-...) ou Kaamelott, jouer avec l'image stéréotypée du casque à cornes viking signale sa permanence et sa popularité ${ }^{14}$.

À ces deux principaux types de « séries médiévales », que l'on pourrait donc qualifier d' « historiques » et de « non historiques », nous aurions sans doute pu ajouter quelques productions contenant des références plus subtiles, moins immédiatement intelligibles, à des œuvres ou des événements de la période médiévale : pensons par exemple à la minisérie The Canterbury Tales ${ }^{15}$, où six contes de Chaucer sont adaptés dans le contexte de l'Angleterre contemporaine, le long de la route qui va de Londres à Cantorbéry ; dans la plupart des épisodes, seuls le titre et quelques références plus ou moins codées, qui sont autant de clins d'œil au spectateur cultivé, suggèrent l'origine médiévale du récit. Mais de telles œuvres, plus rares, n'ont pas fait l'objet d'une attention aussi soutenue, et ce numéro traite donc pour l'essentiel des productions qui prétendent s'inscrire dans un Moyen Âge historique ou de fantasy. Il convient d'ailleurs, bien souvent, de traiter ensemble ces deux types d'œuvres : c'est ce qu'ont fait Florian Besson et Simon Hasdenteufel pour leur analyse du rapport à l'espace, dont les ficelles sont somme toute assez constantes, qu'il s'agisse d'œuvres à prétention historique comme la série Marco Polo (2014-2016) ou de productions largement humoristiques comme la française Kaamelott; de même, Roberto Facchini et Davide Iacono d'une part, Pierre Brice-Stahl

13. M. PAGÈS et K. KINANE, « Introduction... », p. 6.

14. Il en est ainsi des nombreux clichés médiévalistes analysés dans les diverses contributions de F. BESSON et J. BRETON éd., Kaamelott, un livre d'histoire, Paris, 2018. L'ouvrage de L. D'ARCENS, Comic Medievalism. Laughing at the Middle Ages, Cambridge, 2014, aborde assez peu les séries télévisées mais plusieurs de ses observations peuvent leur être appliquées : voir p. 163-168 sur le traitement comique de l'incontournable casque à cornes viking.

15. BBC One, 2003. 
de l'autre, montrent comment les signes d'un « Nord médiéval » ou d'un « médiévalisme boréal » peuvent être déclinés dans Vikings autant que dans Game of Thrones.

Cette dernière série, devenue elle-même en quelques années un véritable «classique » des études médiévalistes ${ }^{16}$, s'avère typique de ce rapport complexe aux corpus de référence, textuels ou autres, qui donnent accès aux signes de médiévalité et permettent de mieux comprendre ce qui «fait médiéval ». Les huit saisons de cette série sont principalement adaptées de l'œuvre romanesque de George R. R. Martin, qui revendique lui-même plusieurs inspirations médiévales : l'une proprement historique, puisqu'il puise dans l'histoire de l'Angleterre au XV $\mathrm{X}^{\mathrm{e}}$ siècle - en particulier la guerre des Deux-Roses ; l'autre plus littéraire, avec pour modèles Les Rois maudits de Maurice Druon, mais aussi tout l'héritage de la fantasy anglophone, qui n'est pas seulement médiévalisante ${ }^{17}$. Il est vrai que la série télévisée multiplie les signes de médiévalité, parmi lesquels les dragons, là encore, occupent une place centrale ; ces signes proviennent de l'œuvre de Martin, mais aussi de toute une imagerie qui la dépasse largement. Si aucune des études ici rassemblées ne porte exclusivement sur Game of Thrones, plusieurs d'entre elles l'incluent naturellement dans leur corpus.

L'étude des séries d'inspiration médiévale dévoile donc le plus souvent une grande variété de sources, qui s'inscrivent dans une logique de cross-médialité, c'est-à-dire de dialogue entre de nombreux supports de discours médiévalistes ${ }^{18}$. Il en résulte que, si les showrunners, scénaristes et réalisateurs appréhendent parfois le Moyen Âge à travers les sources primaires et les travaux des historiens ou des archéologues, ils se référent aussi, et sans doute bien plus, à des productions culturelles plus tardives, elles-mêmes dominées par quelques grands passeurs tels que William Shakespeare, Walter Scott, John R. R. Tolkien ou encore Terence H. White - pour ne citer que quatre noms qui, par leur influence sur la culture

16. Bien que le premier épisode ait été diffusé il y a moins de dix ans sur la chaîne câblée $\mathrm{HBO}$, la bibliographie est déjà devenue imposante, en anglais et dans bien d'autres langues : voir par ex. S. ROLET, Le Trône de fer ou le pouvoir de sang, Tours, 2014 ; F. BESSON, C. KIKUCHI et C. TROADEC, «Les Moyen Âge de Game of Thrones », Cahiers de recherches médiévales et humanistes, 28 (2014), p. 479-507 ; R. FACCHINI, "I watch it for historic reasons." Representation and Reception of the Middle Ages in A Song of Ice and Fire and Game of Thrones », Práticas da História, 4 (2017), p. 43-73 ; C. LARRINGTON, Winter Is Coming. The Medieval World of Game of Thrones, Londres, 2016 (trad. fr., Winter is coming. Les racines médiévales de Game of Thrones, Paris, 2019).

17. Trompés par le caractère ouvertement médiévalisant de la série, certains auteurs ont cherché dans la mythologie scandinave l'origine des fameux Marcheurs blancs, alors que Martin s'est ici visiblement inspiré des wendigos (ou windigos) des légendes algonquiennes : voir S. Smallman, Dangerous Spirits. The Windigo in Myth and History, Toronto, 2014, p. 73.

18. Sur le rôle essentiel de la cross-médialité dans les fictions sérielles contemporaines, voir M. LETOURNEUX, Fictions à la chaîne... 
anglophone de notre temps, jouent un rôle central dans la représentation du Moyen Âge que véhiculent les séries ${ }^{19}$. De même, l'important travail de reconstitution que suppose la production d'une série, et qui concerne aussi bien les vêtements, les objets et même les gestes de la vie quotidienne, que la parole, la musique et tout ce qui relève de la bande-son, se fonde régulièrement sur la reprise de schèmes iconographiques ou sonores élaborés pour l'essentiel au cours de la période romantique. Pourtant, les concepteurs des séries aiment faire appel à des experts et des spécialistes aux compétences malheureusement inégales. Le résultat n'est pas forcément ridicule, et les questions concrètes qu'ils se posent, notamment en ce qui concerne la culture matérielle, touchent à des champs historiques aujourd'hui en plein essor. Il en est ainsi de l'étude des paysages sonores que propose ici Marie-Emmanuelle Torres.

À travers les articles que l'on va lire, c'est donc un autre Moyen Âge qui se révèle. Un Moyen Âge reconstruit, transformé, fantasmé. Un Moyen Âge qui n'a pas existé, sans doute, mais qui est aussi le seul que le grand public a vu. Car ces séries, populaires et largement diffusées, ont fourni et continuent à nourrir un répertoire d'images, de thèmes et de sons qui constituent désormais pour tous le « vrai Moyen Âge », le Moyen Âge vivant, à la fois coloré et sonore, plaisant aussi, avec lequel l'étude des documents d'archives a bien du mal à rivaliser. Pour les médiévistes, connaitre et comprendre ce Moyen Âge de fantasy ne constitue donc pas seulement un aimable divertissement : c'est aussi un véritable enjeu.

\footnotetext{
Alban Gautier - Université de Caen Normandie, Centre Michel de Boüard CRAHAM (UMR 6273)

Laurent Vissière - Sorbonne Université, Centre Roland Mousnier (UMR 8596)
}

19. Concernant l'influence omniprésente de l'œuvre de Terence H. White (The Once and Future King, 5 vol., 1938-1977) sur toutes les adaptations «arthuriennes » de la seconde moitié du XX $\mathrm{X}^{\mathrm{e}}$ siècle, voir en dernier lieu J. BRETON, Le Roi qui fut et qui sera. Représentations du pouvoir arthurien sur petit et grand écrans, Paris, 2019. 
